\title{
Arbor
}

\section{Lecciones aprendidas en el conflicto de los Balcanes}

\section{Angel de Cózar López}

Arbor CLXXI, 674 (Febrero 2002), 283-299 pp.
Condensado de documentos no clasificados procedentes del Mando Aéreo de Combate del Ejército del Aire

El autor ha sabido aquí extraer y condensar, a partir de documentos no clasificados del Mando Aéreo de Combate, una sintesis de las deficiencias encontradas en las operaciones desarrolladas para solucionar el conflicto de los Balcanes, en áreas tan diversas como:

- Gestión de Crisis

- Comunicaciones y Sistemas de Información

- Cooperación Cívico-Militar

- Generación de Fuerzas

- Selección de Objetivos

- Inteligencia...

En todo el artículo se agradece el esfuerzo del autor por huir de tecnicismos, a fin de lograr que éste sea no sólo de interés para los profesionales, sino también para el gran público.

Es importante destacar las conclusiones sobre las carencias de la OTAN europea en ciertas capacidades esenciales para llevar a cabo eficazmente una operación militar en el escenario estratégico actual.

\section{Introducción}

Como en todo conflicto, las partes enfrentadas procuran sacar provecho de sus acciones, tanto de las acertadas como de las erróneas, para in- 
crementar la eficacia de sus Fuerzas Armadas. De esta forma se analizan muchos aspectos que abarcan, desde la toma de decisiones en el ámbito político, hasta el momento en el que esa decisión queda reflejada en el campo táctico o estratégico.

El estudio de las acciones al que se refiere el párrafo anterior puede llevar a creer que en el próximo conflicto habrá que emplear ciertas estrategias o tácticas que fueron deducidas del precedente. Esto puede tener y de hecho ha tenido, consecuencias irreparables. Es arriesgado, ya que el conflicto que puede aguardarnos posiblemente no se parezca al que ha sido objeto de estudio, pero no existe otra vía para aprender. Por eso, se debe estar constantemente alerta para ir detectando los nuevos desafíos que nuestra civilización pudiera tener que afrontar, e ir preparando estrategias, tácticas y unas fuerzas adaptadas para satisfacer las exigencias que un próximo conflicto demande.

$\mathrm{El}$ artículo se estructura en aquellas áreas en que se han detectado deficiencias que pueden ser corregidas sin grandes esfuerzos. Se ha consultado informes de organismos, cuarteles generales, organizaciones y unidades, participantes en el conflicto. No se ha descendido a detalles específicos. Se ha hecho hincapié en no abusar de las siglas, pero no cabe duda que cada vez éstas se van integrando más en nuestro entorno.

\section{Antecedentes}

La experiencia de OTAN en Operaciones de Mantenimiento de Paz se ha caracterizado por un estrecho control político sobre cualquier tipo de operación militar, con todas y cada una de las naciones siguiendo sus propias políticas nacionales e intentando determinar cual sería la mejor respuesta a la crisis en curso. Para las operaciones OTAN, diecinueve países deben exponer sus puntos de vista y resolver sus diferencias al objeto de alcanzar el consenso que permita llevar a cabo una determinada respuesta. Por su propia naturaleza, este no es un proceso que permita a la Alianza la elaboración de una estrategia global o de conjunto a largo plazo, promoviendo en consecuencia una estrategia por etapas que demuestra cohesión, pero al mismo tiempo resulta políticamente frágil.

Tras las operaciones «Tormenta del Desierto» (liberación de Kuwait) «Deliberate Force»(Bosnia Herzegovina, mayo-agosto 1995) y la que nos ocupa, se ha vuelto a poner de manifiesto el estrecho control político al que se han visto sometidas las operaciones y la débil unión entre los miembros de la Alianza en cuanto a sus compromisos en las operaciones militares en Kosovo. Declaraciones públicas realizadas por distintos jefes 
Lecciones aprendidas en el conflicto de los Balcanes

de gobierno al comienzo de la crisis, así como puntos de vista contradictorios referentes a la conveniencia de llevar a cabo operaciones militares ofensivas, limitaron las posibles líneas de actuación de OTAN para resolver la crisis.

Los condicionantes políticos provocaron que el planeamiento de las operaciones se realizase de una manera fragmentada y reactiva según la evolución de la crisis. La falta de autorización por parte de OTAN para elaborar un plan de campaña global, desembocó en un planeamiento por etapas que no logró la sincronización necesaria en las operaciones militares conjuntas. El resultado ha sido que algunos principios militares básicos de Mando y Control, tales como la Unidad de Mando y Esfuerzo, no fueron del todo observados. La dificultad de OTAN a la hora de alcanzar decisiones políticas en el momento oportuno, ha sido desde el punto de vista militar (retrasos en las Órdenes de Activación de los planes operativos), un constante desafío a la hora de realizar los despliegues en el tiempo previsto y un riesgo operativo adicional. Un planeamiento militar previo podría involuntariamente demostrar una cierta voluntad política y, al mismo tiempo, constituir para el Consejo del Atlántico Norte un asesoramiento militar riguroso, exhaustivo y comprensible.

A continuación se analizan brevemente, encuadrados en áreas, los aspectos más destacables relacionados con la doctrina, política y procedimientos de OTAN que podrían ser mejorados, así como las carencias detectadas, tanto en personal cualificado como en capacidades operativas.

\section{En el área de planes}

Las directrices político-militares recibidas para iniciar el planeamiento de las operaciones, habrían sido mucho más claras y comprensibles, si su formato y contenido se hubiera ajustado al que describe el documento del Comité Militar (MC-133), bajo la denominación de «Directiva Inicial». Por otra parte, y de acuerdo con el citado documento, la autoridad de planeamiento no puede ser delegada por debajo del Comité de Planes de Defensa del Consejo del Atlántico Norte (NAC/DPC) o del Comité Militar, lo cual limita la capacidad de los Comandantes responsables para llevar a cabo el planeamiento de las operaciones en respuesta a situaciones que requieren una rápida intervención. La última revisión del MC-133 (versión MC-133/3) en período de consultas y comentarios, garantiza una cierta autoridad a los Comandantes responsables para realizar un planeamiento avanzado, lo cual, si el documento es finalmente aprobado, ayudaría a solventar la situación actual. 
El Proceso de Planeamiento Operativo OTAN (OPP), tal y como está descrito en las Guías de Planeamiento Operativo (GOP), no fue seguido por los responsables del planeamiento a nivel estratégico, pero si lo fue en los niveles operativo y táctico. El planeamiento en estos niveles fue minucioso, integrado y adecuado a cada situación, aunque careció de directrices claras del nivel estratégico. El planeamiento a nivel nacional nunca estuvo bien integrado con el correspondiente de la Alianza, siendo necesarias una mayor transparencia y coordinación entre los responsables nacionales y los de OTAN.

\section{Asesoramiento militar}

No existe en el Cuartel General Supremo Aliado (SHAPE) un proceso formal que desarrolle un análisis que sirva de base para que su Comandante (SACEUR), asesore militarmente a las autoridades políticas. En lugar de realizar su propio análisis estratégico, SHAPE confió plenamente en las valoraciones operativas realizadas por el Mando Regional Sur (AFSOUTH). En la actualidad no hay ni en el ámbito del Comité Militar, ni en el del Mando Estratégico (SC) ningún tipo de directiva, procedimiento o formato establecido en los que apoyarse para proporcionar asesoramiento militar al nivel político. A pesar de que en gran medida la visión y aproximación estratégica de la OTAN sobre Kosovo dependía del asesoramiento militar, SHAPE no ocupó su puesto en el Grupo de Coordinación Política (PCG) y confió en gran parte en el Estado Mayor Internacional (IMS) para exponer el punto de vista de SACEUR.

La participación de SHAPE en el Grupo de Coordinación Política, donde los condicionantes políticos y militares se traducen en instrucciones y directivas para su aprobación por el Consejo del Atlántico Norte (NAC), habría aumentado no solo la capacidad de SHAPE para influir en el proceso, sino también el conocimiento de determinados aspectos políticos que potencialmente podían afectar al posterior planeamiento militar.

\section{Desarrollo de las opciones militares}

Las autoridades políticas OTAN ordenaron a las militares desarrollar una serie de "posibles opciones militares individuales», para una posible intervención militar en Kosovo. Esta orden no constituyó la asignación oficial de una tarea de planeamiento realizada a través de una Directiva Inicial, sino que se llevó a cabo mediante peticiones de asesoramiento mi- 


\section{Lecciones aprendidas en el conflicto de los Balcanes}

litar sobre aspectos específicos y concretos. En total se desarrollaron y valoraron dieciocho opciones diferentes, en función de su probabilidad, coste y eficacia, desde el punto de vista militar. El desarrollo posterior de estas opciones debería haberse realizado en el contexto de una campaña secuencial y sincronizada que apoyase una estrategia militar. Esto no sucedió así, y en su lugar OTAN encargó a SHAPE el desarrollo individual de opciones que pudiesen ser puestas en marcha en respuesta a acontecimientos específicos en Kosovo. Esta actitud aumentó significativamente la carga de trabajo de los responsables del planeamiento militar, situándolos en una postura meramente reactiva y fragmentando el planeamiento posterior.

\section{Directrices Político/Militares y tareas asignadas al Mando Estratégico}

Las directrices políticas al Comandante Estratégico para el desarrollo de los planes no fueron realizadas de una manera clara, integrada y coherente. No existió una única Directiva Inicial, la cual debía proporcionar los objetivos políticos y militares que sirven de base para la elaboración de un plan militar integrado. En su lugar, las directrices se recibieron por etapas, a menudo muy específicas en determinadas opciones (sólo empleo de medios aéreos) y de formas muy diversas. Al final, los resultados han demostrado que las directrices recibidas han sido adecuadas, aunque para los responsables del planeamiento ha constituido un esfuerzo adicional el integrar y localizar estas fragmentadas directrices provenientes de distintas fuentes.

\section{Directrices estratégicas proporcionadas a los mandos subordinados}

Responsables del planeamiento en el ámbito estratégico y operativo, así como diversos altos cargos del Mando Aliado, se quejan de la falta de detalle y claridad en las directrices recibidas. Esto se podría haber evitado si el Comandante Estratégico hubiese desarrollado su propia directiva de planeamiento. La visión estratégica e intenciones de SACEUR sobre Kosovo no estaban reflejadas en documento alguno, y de acuerdo con los responsables de planeamiento y otros altos cargos, permanecieron sin estar claras pese al contacto diario a través de videoconferencia.

SHAPE todavía no había definido claramente a los responsables del planeamiento operativo los objetivos militares a alcanzar, cuando la Alianza desplegó sus fuerzas en el teatro y comenzaron las operaciones. 


\section{Preparativos militares previos a una crisis}

Con anterioridad a Abril de 1998, los responsables del planeamiento militar en OTAN no fueron autorizados a realizar un plan avanzado en previsión de una eventual intervención en Kosovo. En concreto, responsables de planeamiento en el Mando Regional Sur (AFSOUTH), solicitaron autorización en Febrero de 1997 para iniciar un estudio previo sobre Kosovo y les fue denegado. Esta restricción puso a los responsables del planeamiento en una posición reactiva, con poca preparación previa, cuando finalmente, en Abril de 1998, tuvieron que hacer frente al planeamiento asignado.

El planeamiento operativo depende de una serie de datos que, en muchos casos, requieren bastante tiempo para su obtención o desarrollo. Los responsables de planes en las operaciones de Kosovo se vieron forzados a realizar estudios, valoraciones y a obtener datos para el planeamiento, de forma simultánea a la elaboración de los planes. Esto provoca siempre en el mejor de los casos, una dilatación en el tiempo del citado proceso. Los estudios y valoraciones previas proporcionarían una mejor capacidad de respuesta al anticipar determinadas tareas críticas del planeamiento. Últimamente se recoge en la documentación correspondiente, la posibilidad de que los Comandantes Estratégicos puedan llevar a cabo actividades de planeamiento preparatorias, siempre y cuando los informes de inteligencia así lo aconsejen.

\section{Determinación de los Centros de Gravedad ${ }^{1}$}

Los Centros de Gravedad no fueron identificados de una manera consistente en el planeamiento a nivel estratégico realizado por SHAPE. El Mando Regional Sur (AFSOUTH) dedicó un considerable esfuerzo a su identificación para asegurar un punto de vista común de todos los componentes del planeamiento conjunto. SHAPE identificó los Centros de Gravedad para la Operación Aérea por Fases (PAO). Sin embargo, hubo diferencias entre los planes estratégicos (SHAPE) y operativos (AFSOUTH). Estas diferencias no fueron nunca subsanadas durante el proceso de planeamiento y tuvieron repercusiones operativas durante la ejecución. El debate sobre si la Selección de Objetivos debía centrarse en el sistema de Mando y Control e infraestructuras de la Antigua República de Yugoslavia o en los efectivos de su $30^{\circ}$ Ejército, debería haber sido objeto de análisis por el Estado Mayor durante las primeras etapas del planeamiento de las Operaciones Aéreas, y la decisión tomada por SACEUR, convenida por el Mando Regional Sur y los Mandos Componentes. 


\section{Planeamiento a nivel estratégico}

El planeamiento de las operaciones por SHAPE y AFSOUTH fue en gran parte redundante, con muy pocas diferencias en alcance y contenido de sus respectivos documentos de planeamiento. El documento del Comité Militar, MC 53-2, establece como una de las responsabilidades de SACEUR el planeamiento de ámbito estratégico. En general, SHAPE pareció hacer frente a esta responsabilidad proporcionando a las autoridades políticas versiones ligeramente modificadas de los planes y conceptos elaborados por el Mando Regional Sur. Aunque esto facilitó la oportuna aprobación por el Consejo del Atlántico Norte (NAC) de los Planes Operativos, también impulsó a SHAPE a prestar gran parte de su atención a aspectos operativos y algunas veces incluso tácticos.

\section{Proceso de planeamiento de las operaciones}

El Proceso OTAN de Planeamiento de las Operaciones (OPP), tal y como se establece en los documentos apropiados, ha demostrado ser, en líneas generales, eficaz, y fue utilizado correctamente en su aspecto operativo por el Mando Regional Sur. En el nivel estratégico, este proceso no fue fielmente seguido por SHAPE, estuvo fragmentado, realizándose el planeamiento terrestre, naval y aéreo, por células independientes, existiendo poca interacción entre ellas; no se creó formalmente un grupo de planeamiento de operaciones, que asegurase que otras áreas funcionales de relevancia como las de Cooperación Cívico Militar (CIMIC), Operaciones de Información, Aspectos Jurídicos, Logística, etc., estuviesen integradas en un único esfuerzo de planeamiento.

\section{Planeamiento paralelo USA}

Las operaciones en Kosovo han puesto de manifiesto la falta de acuerdos para integrar el planeamiento realizado de forma concurrente por OTAN y los Estados Mayores nacionales. Dada la preponderancia de medios aéreos de los EEUU asignados a las Operaciones Aéreas (70\%), es comprensible que por su parte hubiera un gran interés en el planeamiento de OTAN. En consecuencia, los EEUU crearon un Cuartel General de una Fuerza Expedicionaria Conjunta Nacional, el cual se formó en el Mando Regional Sur (AFSOUTH) y cuyo núcleo lo constituía personal norteamericano destinado en Europa. Sin embargo, la realidad demostró que la puesta en funcionamiento de la mencionada fuerza detrajo personal USA asignado tanto al Mando Aéreo del Sur (AIRSOUTH), como a 
STRIKEFORSOUTH (Fuerza Aeronaval norteamericana que forma parte del Mando Sur de OTAN), degradando notablemente las capacidades de estos dos Organismos.

El planeamiento llevado a cabo por la Fuerza Expedicionaria fue independiente del correspondiente OTAN, aunque al más alto nivel sí hubo conocimiento de ambos procesos de planeamiento, debido a la doble función desempeñada por los máximos responsables de ambas organizaciones. En el ámbito de los Estados Mayores no existió ningún tipo de comunicación que permitiera coordinar y sincronizar ambos procesos. Evidentemente, esta falta de sincronismo y coordinación trajo como consecuencia el desconocimiento por parte de OTAN de los planes norteamericanos, ya que los de la Alianza eran suficientemente conocidos y accesibles.

De la misma manera, el proceso de Selección de Objetivos fue enteramente norteamericano realizado a través de una Oficina de Coordinación Conjunta (USA), lo que impidió que OTAN tuviera posibilidad de influir en el mismo y menos aún las naciones. Los objetivos se aprobaban en Washington.

\section{Capacidades en el planeamiento de las operaciones}

La organización del planeamiento en SHAPE está estructurada de forma que los planes carecen de integración y carácter conjunto. El planeamiento de las operaciones aéreas, terrestres y navales se realizó de manera aislada. No se creó un Grupo de Planeamiento de Operaciones que dirigiera y controlara todo el proceso, de forma que asegurara la integración de todas y cada una de las áreas funcionales implicadas. Por otro lado, la dotación de personal en la Organización para Gestión de Crisis (CMO) de SHAPE, el Centro de Operaciones Conjuntas, resultó ser insuficiente en cuanto a número y desequilibrada, ya que la gran mayoría de sus miembros pertenece al Ejército de Tierra, con escasa o nula representación Naval y Aérea. En este Cuartel General la falta de personal y entrenamiento resultó tan acusada, que su contribución en el planeamiento de las operaciones aéreas fue prácticamente nula, incidiendo de manera muy negativa en el desarrollo general de las operaciones llevadas a cabo durante la campaña.

\section{Generación de fuerzas}

El Proceso de Generación de Fuerzas, en general, aseguró una correcta identificación de las fuerzas necesarias y facilitó la coordinación 
con las Naciones para cubrir estas necesidades. Sin embargo, se detectó una falta de procedimientos formalmente establecidos que aseguraran la normalización de todo el proceso y tampoco existía un acuerdo generalizado sobre el ámbito de aplicación y las actividades que abarcan el mismo. En cuanto a la identificación de fuerzas y coordinación de las contribuciones nacionales, en algunas áreas las Naciones aportan más fuerzas de las requeridas, aumentando las necesidades de elementos de apoyo y dejando sin cubrir otras áreas funcionales que habían sido identificadas como realmente necesarias. En general, SHAPE nunca rechaza las contribuciones de las Naciones aunque sean en exceso sobre las necesidades inicialmente previstas, o aunque respondan a una capacidad no identificada como necesaria.

Los procedimientos OTAN para la activación de fuerzas están directamente vinculados al proceso de aprobación de los Planes Operativos. Actualmente, una Orden de Activación (ACTORD) debe ser emitida para que el proceso de activación de la fuerza pueda tener lugar. En consecuencia, los retrasos políticos en la autorización de las ACTORD para los correspondientes Planes, motivaron demoras en la Transferencia de Autoridad (TOA) de las fuerzas, lo cual tuvo su incidencia en las operaciones.

Según la normativa OTAN en vigor, SACEUR es responsable de establecer procedimientos para la generación de fuerzas que aseguren la oportuna creación e integración de fuerzas y capacidades. Sin embargo, desde que el término «Generación de Fuerzas» fue empleado por primera vez, a finales del año 1995, aún no ha sido formalmente definido. Por otra parte, no existe un acuerdo generalizado sobre el conjunto de actividades que dicho término engloba. El concepto empleado en SHAPE para generación de fuerzas está referido exclusivamente al proceso mediante el cual el Estado Mayor identifica y coordina las necesidades de fuerzas. Sin embargo, la generación de fuerzas para las operaciones en Kosovo ha puesto de manifiesto que es preciso realizar más actividades si se pretende que las fuerzas estén operativamente listas y disponibles en el momento oportuno, para su utilización por los Comandantes OTAN. Consecuentemente, una vez identificadas las necesidades de fuerzas y coordinadas las contribuciones nacionales, las fuerzas tienen que ser activadas, organizadas, proyectadas al teatro de operaciones y transferidas bajo la autoridad del Comandante OTAN.

Todas estas actividades están recogidas, parcialmente y por separado, en distintos documentos y directivas OTAN, pero no están armonizadas entre sí, y no existe un único documento que las vincule a todas ellas dando un sentido más amplio al concepto de Generación de Fuerzas. 


\section{Angel de Cózar López}

\section{Procedimientos para generación de fuerzas}

Los procedimientos para la generación de fuerzas, utilizados en las operaciones de Kosovo, fueron en general eficaces a la hora de identificar y cubrir las necesidades de fuerzas. El desarrollo de una Identificación de Necesidades (SOR), para cada concepto de las operaciones, demostró ser un medio efectivo para coordinar las necesidades de fuerzas con las $\mathrm{Na}$ ciones. No obstante, no existen procedimientos establecidos que aseguren que las contribuciones nacionales se integren de una manera efectiva en el desarrollo del plan y normalmente SHAPE no rechaza fuerzas que con carácter adicional ofrecen las naciones, incluso una vez cubiertas las necesidades, como se ha mencionado anteriormente.

SHAPE reflejó las variaciones que se producían en las contribuciones nacionales en una serie de revisiones a las SOR. En algunas ocasiones, esta práctica dio como resultado la aparición de más de diez versiones diferentes de una SOR para un Plan. Por otra parte, las prioridades en el despliegue de fuerzas no fueron establecidas nunca, y las SOR tampoco reflejaron cuándo las fuerzas debían estar operativamente listas para actuar. Todo ello provocó la imposibilidad de elaborar un plan de despliegue multinacional, coherente e integrado, que respondiera a las necesidades operativas del Comandante sobre el terreno.

\section{Nación Líder en el proceso de generación de fuerzas}

El proceso de generación de fuerzas fue dirigido por una Nación Líder (LN) sin contacto alguno con SHAPE. Esto provocó confusión y múltiples malentendidos, al ser contradictorios los puntos de vista de SHAPE y la Nación Líder. Este término ha sido utilizado con anterioridad en el ámbito de apoyo logístico.

\section{Identificación de las necesidades}

El proceso de Identificación de Necesidades queda recogido en un documento, (State of Requirements SOR) por el que el Mando Estratégico, de acuerdo con el Comandante de la Fuerza designado, solicita a las naciones las fuerzas que estima necesarias para la operación. El proceso de identificación de las fuerzas para las operaciones en Kosovo fue fragmentado y complicado debido a la falta de existencia de un Plan de Campaña único e integrado. Como consecuencia del planeamiento fragmentado, paralelo, y en muchos casos específico, los distintos SOR elaborados carecieron de la naturaleza conjunta e integración necesaria que debían 
caracterizar a una operación OTAN de esta magnitud. Fue difícil racionalizar y equilibrar las necesidades de fuerzas para los distintos planes, incrementándose los riesgos de redundancias en algunas áreas y las carencias en otras e incluso produciendo duplicidades.

\section{Capacidades deficitarias}

En general, el proceso de generación de fuerzas proporcionó las capacidades identificadas en los documentos SOR, salvo en el caso de algunas áreas críticas, cuyas carencias son una constante en la mayoría de las fuerzas OTAN. En general, las carencias más significativas detectadas durante las operaciones de Kosovo son las siguientes:

- Estructuras y Elementos de Apoyo Logístico.

- Policía Militar.

- Unidades Especializadas Multinacionales.

- Unidades de Vigilancia y Control Aéreo.

- Armamento y Plataformas Aéreas de Combate y de Apoyo al Combate Avanzadas.

- Helicópteros de Ataque y Transporte.

- Comunicaciones Seguras.

- Sistemas/estructuras de Mando, Control e Información.

En detalle, y en palabras del propio General Clark (SACEUR en aquel momento), las carencias más importantes de las naciones OTAN son las siguientes:

- Vehículos a Control Remoto, para reconocimiento y asesoramiento de daños causados, en tiempo real.

- Medios de Combate electrónico, para operaciones de inteligencia electrónica, y supresión de defensas.

- Armamento de precisión, para reducir esfuerzos al batir un objetivo y reducir daños colaterales.

\section{Generación de fuerzas de apoyo}

Las contribuciones nacionales de fuerzas de combate cubrieron siempre las necesidades requeridas, pero como sucedió en IFOR, las fuerzas de Apoyo al Combate resultaron un requisito muy difícil de satisfacer debido al insuficiente número de aportaciones. En particular, las naciones han estado poco dispuestas a proporcionar helicópteros, ingenieros, policía militar y transporte de tropas para apoyar a unidades distintas de las propias. Las carencias de este tipo de unidades, ya detectadas en 1997, se intentaron solucionar estableciendo prioridad a las necesidades de fuerzas 
de Apoyo al Combate, para una unidad tipo Brigada. Sin embargo, no todas ellas tienen las mismas capacidades ni están igualmente dotadas. Dependiendo de la Nación que constituía el armazón y soporte de la Brigada, estas carencias fueron solventadas en mayor o menor medida.

\section{En el área de inteligencia}

El área de inteligencia en OTAN se encuentra en una situación precaria. Prácticamente, todos los recursos disponibles son los que aportan las naciones. El choque permanente entre las inteligencias nacionales con la general de la Alianza, no está resuelto. Resulta una organización excesivamente fragmentada, inadecuada por los problemas de compatibilidad que presentan los distintos sistemas nacionales y la falta de transparencia que en determinadas circunstancias se produce al tratarse de temas de gran sensibilidad. Todo ello da como resultado una organización absolutamente ineficaz. Durante la crisis de Kosovo, el Centro de Análisis Conjunto norteamericano, con sede en el Reino Unido, fue el único centro de inteligencia utilizado para apoyar las operaciones.

\section{En el área de operaciones}

\section{Mando y control}

El Mando y Control fue una de las áreas más problemáticas durante toda la operación. A pesar de que el Plan «Allied Force» establecía claramente la cadena de mando OTAN, acordada por todos los países en el Consejo del Atlántico Norte (NAC), durante la conducción de las operaciones la cadena de mando de los EEUU tuvo absoluta prioridad. El empleo de una cadena de mando nacional, de acuerdo con la política OTAN, es aceptable siempre que complemente los medios aliados disponibles, pero no lo es si funcionando en paralelo complica el proceso y dificulta la coordinación.

El minucioso control político y el gran interés mostrado por los medios de comunicación durante la operación, provocó, en algunos casos, que la atención de los cuarteles generales del más alto nivel estuviera más pendiente de las circunstancias e incidentes que tenían lugar a nivel táctico, que de elaborar y coordinar la estrategia y planeamiento a largo plazo. 


\section{Reglas de enfrentamiento}

El proceso de elaboración y aprobación de las Reglas de Enfrentamiento (ROE) se vio dificultado por la carencia de asesores jurídicos en todos los niveles, aunque fue particularmente grave en el Cuartel General de OTAN donde la aprobación de las Reglas de Enfrentamiento sufrió retrasos tanto en el momento de la sanción de los planes operativos, como cuando se remitían peticiones de Aprobación de Reglas específicas.

Las ROE para las operaciones aéreas estuvieron, en general, en consonancia con las circunstancias, y fueron evolucionando con los cambios de la situación. Sin embargo, en el caso de las fuerzas terrestres y navales, inicialmente no se editó ningún tipo de ROE, al asumir que se iba a operar en condiciones de paz.

\section{Organización para la gestión de crisis}

El actual Centro de Operaciones Conjuntas de SHAPE, se estableció en 1997 como consecuencia de un plan de Gestión de Crisis, elaborado en 1995, pero en la actualidad no cubre las necesidades requeridas para apoyar una operación como la de Kosovo. No existe una base documental sólida que describa el proceso y las actividades que deben desarrollarse. Igualmente, no está definida una plantilla de personal para casos de crisis, lo cual provocó que los distintos puestos de trabajo y funciones a realizar se fuesen identificando y cubriendo a medida que la situación lo requería.

\section{Selección de objetivos y asesoramiento de daños}

La operación «Allied Force» ha puesto de manifiesto que OTAN carece de doctrina, normativa y organización en las áreas de Selección de Objetivos y en la de Asesoramiento de Daños causados. En consecuencia, en ambas disciplinas la dependencia de los medios de EEUU fue total, y el acceso a la información limitado a la que se proporcionaba oficialmente.

La Selección de Objetivos, como el Asesoramiento de Daños, constituyen una asignatura muy difícil. A grandes rasgos, consiste en definir «directivas políticas,» transformarlas en «directivas militares» y éstas a su vez en una lista inicial de «objetivos físicos» sobre el suelo, catalogados y priorizados, en cantidad suficiente para toda la campaña, cuya duración hay que estimar correctamente. La Lista de Blancos Priorizada Conjunta (JPTL) disponible al comienzo de la campaña, se terminó el cuarto día de las operaciones. Los últimos grandes ejercicios OTAN (Strong Resolve 
98) o aliados (Eolo-98 y 00), demuestran hasta qué punto las naciones y organizaciones son deficitarias e incapaces en este área.

\section{En el área de la logística}

La doctrina OTAN para apoyo logístico ha demostrado ser muy eficaz para los responsables del planeamiento logístico. En general, la utilización de un concepto logístico global aplicado a las operaciones en Bosnia y Kosovo ha sido efectivo, aunque no se aplicaron todos los preceptos del apoyo logístico multinacional. En su conjunto, el apoyo logístico fue proporcionado principalmente a través de canales nacionales, complementado por el apoyo prestado por las Naciones Anfitrionas (Host Nation) y Naciones Líder (Lead Nation) según los casos.

Como consecuencia de la Operación Joint Guarantor, se ha puesto de manifiesto que el concepto de Nación Líder, contemplado en la doctrina para apoyo logístico, no está suficientemente detallado, siendo necesaria una definición más precisa de sus funciones, responsabilidades y tareas para asegurar que actúa de acuerdo con la doctrina OTAN. El suministro de carburante se ha visto negativamente afectado por la reducida capacidad del Oleoducto Centro Europeo (Central European Pipeline System). Las fuerzas navales han sufrido igualmente la escasez de buques nodriza para suministro de combustible. En el área de Ingenieros, la falta de personal ha sido una constante en todos los Cuarteles Generales.

El despliegue de fuerzas en el teatro puede considerarse satisfactorio, en el sentido de que éstas llegaron a sus destinos a tiempo. Sin embargo, las incertidumbres políticas comprimieron los tiempos de planeamiento, y el intercambio de información entre OTAN y las naciones no estaba inicialmente autorizado. Ante esta situación, algunas naciones decidieron enviar sus fuerzas antes de la recepción de la orden de activación correspondiente. Gracias al apoyo prestado por las Naciones Anfitrionas (Albania y Macedonia), los problemas surgidos durante la recepción, estancia, movimiento hacia el teatro e integración de todas las fuerzas, fueron convenientemente solventados. El Sistema Aliado para Despliegue y Movimiento (ADAMS) demostró su utilidad como herramienta en la gestión de despliegues durante la fase de evaluación conceptual en el planeamiento, pero no fue eficazmente utilizado durante la ejecución.

\section{En el área de las Comunicaciones y Sistemas}

En general, los medios para Comunicaciones y Sistemas de Información (CIS), puestos a disposición de las operaciones en Kosovo pueden 
considerarse adecuados en cuanto a número. No obstante, se han detectado carencias en algunas áreas que han contribuido a reducir la eficacia global de todo el conjunto. Escasos equipos para Comunicaciones vía Satélite (SATCOM), insuficiente provisión de equipos CIS seguros a nivel de las bases aéreas, ausencia de personal convenientemente cualificado en los Cuarteles Generales y, finalmente, unas relaciones de mando en el área de CIS no definidas claramente, han provocado en algunos casos, duplicidad de esfuerzos y en otros, fallos en la asignación de tareas.

\section{En el área de la Cooperación Cívico Militar}

La doctrina y política OTAN sobre Cooperación Cívico Militar (CIMIC) necesita ser desarrollada con mayor amplitud. Las iniciativas tomadas en 1995 a raíz de las operaciones en Bosnia no se habían concluido cuando comenzó la crisis. El entrenamiento y mentalización en el área de CIMIC es todavía insuficiente en OTAN, no siendo tenida en cuenta suficientemente durante todo el proceso de planeamiento ni durante la ejecución. Al igual que en otros muchos aspectos ya mencionados durante la operación de Kosovo, la postura adoptada fue más reactiva que activa, siguiendo la pauta que determinaban los acontecimientos sin apenas iniciativa.

Asimismo, las carencias de personal especializado en este área han sido evidentes en todos los Cuarteles Generales. Las relaciones con las Organizaciones Internacionales necesitan ser mejoradas y ejercitadas con mayor frecuencia al objeto de aumentar la confianza y el conocimiento mutuo. A nivel interno, OTAN debería revisar su Planeamiento para Emergencias Civiles con la finalidad de hacerlo más fluido y con mayor capacidad de respuesta, pues el actual se ha revelado poco eficaz.

\section{Aspectos Jurídicos}

La falta de recursos humanos y experiencia en el campo del Derecho, en Operaciones de Mantenimiento de Paz (PSO), significó que el desarrollo de mecanismos tales como resoluciones ONU, SOFA, HNS, derechos de tránsito, etc., para Albania y Macedonia no fuesen del todo satisfactorios. OTAN debería establecer para casos de crisis, una célula de asesores jurídicos con una plantilla de personal suficiente para prestar el apoyo legal necesario en este tipo de operaciones y a la vez proporcionar el entrenamiento adecuado a este personal con objeto de facilitar al Comandante OTAN, su proceso de toma de decisión. 


\section{Aspectos Financieros}

Para las operaciones de Kosovo, el nivel de recursos financieros identificados por el Mando Aliado en Europa, fue suficiente para responder a las necesidades operativas sufragadas con fondos comunes OTAN, hasta el final de 1999. A más largo plazo, las necesidades presupuestarias, tanto de KFOR como de SFOR, requerirán fondos adicionales para las fuerzas en el teatro y para los cuarteles generales. El planeamiento para el apoyo de las necesidades de misión, fue complicado al no existir criterios ni principios aprobados por el Consejo del Atlántico Norte, acerca de lo que puede y no puede ser sufragado con fondos comunes. En el futuro, la gestión financiera podría ser mejorada incorporando a la doctrina los procedimientos y experiencias adquiridas de KFOR/SFOR, poniéndolos en práctica durante los ejercicios.

\section{Conclusiones}

Con la desaparición de la amenaza de la Guerra Fría, OTAN reconoció un nuevo escenario estratégico que provocó una revisión a fondo de toda su política y procedimientos. En 1995, las operaciones en Bosnia fueron la prueba de fuego de esta nueva política y procedimientos en el nuevo escenario. Las lecciones aprendidas en IFOR/SFOR, ya ponían de manifiesto carencias en la doctrina, política, procedimientos y recursos disponibles.

En 1999, la crisis de Kosovo puso a prueba una vez más, la política y procedimientos OTAN. Las mismas deficiencias ya detectadas tres años antes, continúan estando presentes en áreas como las de Gestión de Crisis, Comunicaciones y Sistemas de Información, Cooperación Cívico Militar, Generación de Fuerzas, Selección de Objetivos, Inteligencia, etc. En consecuencia, una lección que se debe aprender de las operaciones en Bosnia y Kosovo, es que hasta que no se tomen las medidas correctoras y se apliquen los cambios necesarios, tanto en política como en procedimientos, las lecciones serán únicamente identificadas, y no aprendidas.

A lo largo de toda la crisis de Kosovo, OTAN confió excesivamente en la preponderancia de medios y capacidades de los EEUU, para dirigir y conducir adecuadamente la Operación «Allied Force». Mientras la Alianza parece sentirse cómoda permitiendo a USA desempeñar esta función, los interrogantes acerca del futuro de la Iniciativa sobre Identidad de Defensa y Seguridad Europeas (ESDI) son cada vez mayores. La Operación «Allied Force» ha demostrado claramente que OTAN, en conjunto, tiene 


\section{Lecciones aprendidas en el conflicto de los Balcanes}

la capacidad de acometer cualquier misión que se le pueda encomendar. Sin embargo, la OTAN europea todavía carece de ciertas capacidades esenciales y de la experiencia necesaria para llevar a cabo eficazmente una operación militar en el escenario estratégico actual.

\section{Notas}

1 Por Centro de Gravedad se entiende aquel punto considerado vital a batir o neutralizar, para doblegar la voluntad del enemigo. 